\title{
Review (Narrative)
}

\section{Graphene}

\section{A Promising Candidate for Biomedical Use}

\author{
Eva Clithy, Ph.D.
}

\section{SUMMARY}

As a new two-dimensional nanomaterial composed of $s p^{2}$ carbon atom, graphene has unique electrical, optical and mechanical properties. At present, the research on graphene and its derivatives is mainly concentrated in the field of physics research. The chemical and material science research of graphene is also developing rapidly, and the research work of graphene in biomedicine field has just begun. Graphene oxide is a derivative of graphene, which has a high specific surface area and a rich functional group: the bottom surface contains a hydroxyl group and an epoxy group, and the edge contains a carboxyl group. These functional groups impart good hydrophilicity, dispersibility and biocompatibility to graphene oxide, are easy to modify and functionalize, and combined with their excellent optical properties, make graphene oxide have broad application prospects in the biomedical field. We herein briefly describe the recent advances in graphene, especially graphene oxide, in the biological and medical fields, including targeted drug delivery, cell imaging, bioassays, oncology, and graphene biosafety.

\section{KEYWORDS}

Graphene; Graphene Oxide; Biomedical Materials; Biological Safety

Sci Insigt. 2019; 29(3):52-57. doi:10.15354/si.19.re089

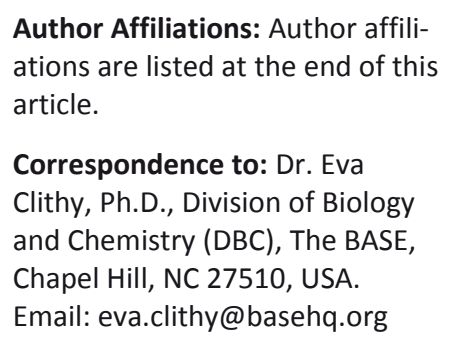

Author Affiliations: Author affiliations are listed at the end of this article.

Copyright (C) 2019 The BASE. This is an open access article distributed under the Creative Commons Attribution License, which permits unrestricted use, distribution, and reproduction in any medium, provided the original work is properly cited. 
RAPHENE is a single atomic layer of closely spaced two-dimensional material with the honeycomb structure formed by the $s p^{2}$ hybridized carbon atoms. Andre Geim and Konstantin Novoselov (1) first successfully prepared and observed graphene by micromechanical stripping of highly oriented pyrolytic graphite (HOPG) in 2004, and both of them shared the 2010 Nobel Prize in Physics.

Due to its special two-dimensional structure of monoatomic layer, graphene has excellent performance in electronics, optics, mechanics and thermals. Graphene has a good application prospects in electronics (flexible electronic materials, high-frequency transistors, logic transistors, etc.), photonics (photodetectors, light modulators, mode-locked lasers/Terahertz signal generator, the optical polarization controller, etc.), composite materials, coatings, energy, and biomedical and other fields (2).

Carbon is one of the basic elements of the composition of living organisms, and the study confirmed that, certain modified graphene does have good biocompatibility, so graphene has a natural advantage in the field of biomedicine. Because real graphene is difficult to achieve a large number of preparations, therefore, the practical application is mostly graphene derivatives, such as a graphene layer (2 to 10 layers), a multi-layer graphene (10 layers or more, but less than $100 \mathrm{~nm}$ or a thickness), Graphene oxide (GO) and reduced graphene oxide (rGO) and the like. At present, graphene and its derivatives have been proven to be applicable to biomedical related fields such as drug gene delivery, composite materials, tumor photothermal therapy, bioimaging, electrochemical biosensors, antibacterial and tissue engineering.

\section{GRAPHENE IN THE FIELD OF BIOMEDICAL APPLICATIONS}

\section{Structural Properties of Graphene}

Graphene, in particular, has a large surface area of GO and has good dispersibility in water. The surface of GO is rich in oxygen-containing functional groups, which can chemically immobilize various drugs and biomolecules on its surface, so it has a good application prospect in the field of drug and gene delivery. In the field of biomedicine, we can use a variety of external means to load a water-insoluble drug into a water-soluble graphene carrier, thereby changing it into a carrier drug with certain water solubility. These include noncovalent modification, $\mathrm{pH}$ regulation, and molecular modification (3).

In addition to being used to deliver drugs, graphene can also be used as a gene vector for gene transfection. The GO surface contains a large number of functional groups such as carboxyl groups and hydroxyl groups, making it easier to obtain a positively charged graphene complex by surface modification for gene transfer transfection related research. Currently, positively charged materials such as polyethyleneimine, chitosan and 1indolylmethylamine have been used to modify graphene and serve as a gene delivery vector.

\section{Application of Mechanical Properties of Graphene}

Since the two-dimensional structural characteristics which layers of carbon atoms, graphene having excellent mechanical properties, the Young's modulus of the unsupported graphene up to $1.0 \mathrm{TPa}$, the intrinsic strength of $130 \mathrm{GPa}$. Thus, the graphene can be used as the respective two-dimensional reinforcing composite materials.

Hydroxyapatite (HA) has similar chemical composition and crystal structure to calcium phosphate in human bone tissue, so it has excellent biocompatibility and is suitable for adhesion, proliferation and osteogenic mineralization of osteoblasts. However, its inherent covalent bond or covalent bond/ion bond coexistence features poor fracture toughness and reduced reliability and service time of HA. The graphene/hydroxyapatite nanorod composite material synthesized by the innate characteristics of graphene can significantly improve the Young's modulus and mechanical properties of hydroxyapatite.Meanwhile, compared with the individual graphene and hydroxyapatite, due to the composite material containing $40 \%$ hydroxyapatite, bone and therefore it exhibits a higher integration, better biocompatibility and excellent bone cell proliferation Induction ability.

As one of the artificial joint materials, ultra-high molecular weight polyethylene (UHMWPE) has good wear resistance, but its wear is still inevitable in longterm use, and the generated abrasives will cause the surrounding bone to dissolve, which will cause aseptic loosening, and then affecting the life of artificial joints. The GO/UHMWPE composite prepared by the intro- 
duction of GO exhibits superior hardness and wear resistance under different lubricating media (4). Polymethyl methacrylate (PMMA) denture base material has excellent simulation aesthetics and biological properties, but its mechanical properties cannot meet the clinical requirements well, mainly in poor wear resistance. Adding a certain amount of GO (mass fraction of about $0.1 \%$ ) can effectively improve its wear resistance under artificial saliva lubrication medium.

\section{Thermal Properties of Graphene}

In view of its excellent photothermal conversion capability in the near-infrared region, graphene is widely used in photothermal treatment of tumors. Graphene modified by polyethylene glycol (PEG) can be enriched into tumor tissue by passive targeting, and applied to photothermal treatment of tumor by its good light absorption ability in the near-infrared region. Meanwhile, hydrazine hydrate reduction modified by PEG produced a $100 \%$ elimination of the tumor tissue under the irradiation of $808 \mathrm{~nm}$ laser light of a magnitude lower than the conventional power (5). In addition to directly killing tumor cells by photothermal therapy, the photothermal conversion effect of graphene is currently widely used in the synergistic treatment of tumors.

\section{Optical Properties of Graphene}

Graphene has a wide application prospect in the field of biological living imaging as an emerging fluorescent imaging material due to its good dispersion stability, biocompatibility and strong fluorescence imaging (6). Graphene itself can fluoresce under the excitation of near-infrared light, and some fluorescent dyes can be connected to graphene by covalent or non-covalent dye to obtain a composite with better fluorescence properties. Therefore, this fluorescent material enables cell imaging and marker detection of tumors.

\section{Electrical Properties of Graphene}

Graphene can promote electron transport, exhibits excellent electrocatalytic behavior for some small biomolecules, and has the advantages of being easy to carry, high sensitivity and low cost (7). Therefore, it has attracted extensive attention in the field of high performance electrochemical biosensors. As a conductive component of the electrode surface and the glucose oxi- dase redox center, graphene can well maintain the biological activity of glucose oxidase and promote the transmission of electronic signals from the active center of the enzyme to the electrode surface. Since the graphene has a large specific surface area, the enzyme can be better supported on the surface thereof, and the glucose oxidase electrochemical biosensor based on the detection has a broad detection range.

The graphene-modified electrode has excellent electrocatalytic activity, and the defects existing at the edge can be used as an active site to increase the electron transfer speed of the detected biomolecule and reduce the electron transfer resistance. The graphenemodified electrode can detect one of the molecules under the condition of multiple biomolecules at the same time, and has the advantages of good separation effect and low detection limit.

In the detection of selected DNA sequences and disease-related variant genes, electrochemical DNA sensors have the advantages of high sensitivity, high selectivity and low cost, and are expected to provide a simple, accurate and inexpensive clinical detection platform. Graphene has a wide potential window and can directly detect nucleic acid molecules with higher oxidationreduction potential.

\section{THE BIOLOGICAL PROPERTIES OF GRAPHENE}

\section{Antibacterial Properties of Graphene}

It was found that both graphene oxide (GO) and reduced graphene oxide (rGO) have excellent antibacterial properties, and GO and rGO films prepared by vacuum filtration also exhibit excellent antibacterial properties. In-depth research results show that the edges of $\mathrm{GO}$ and rGO sheet structures are hard and may cause damage to cell membranes of bacteria such as $E$. coli (8). The GO film was prepared on the surface of the stainless steel substrate by electrophoretic deposition method, and the rGO film was obtained by reduction of hydrazine hydrate, both of which destroyed the integrity of the bacterial cell membrane and exhibited excellent antibacterial properties. The rGO film showed better inhibition of Gram-negative bacteria ( $E$. coli) and Gram-positive bacteria $(S$. aureus) than the GO film, mainly due to the interaction of rGO with bacteria with sharper edges. It has better charge transfer characteristics. It was found 
that the agglomerated $\mathrm{GO}$ and $\mathrm{rGO}$ can be separated from the external environment by capturing $E$. coli, and the bacteria stop growing or even die due to the inability to obtain nutrients efficiently, so GO and rGO show good antibacterial properties (9). By comparison, it is found that the structural properties and the size of the sheet of the graphene-based material affect its antibacterial properties.

Under the same conditions, GO showed the best antibacterial property, followed by rGO, graphite and graphite oxide; large-size GO has more excellent antibacterial properties. At the same time, it is found that the antibacterial property of graphene-based materials is mainly due to the membrane stress caused by the sharp edges of the graphene in contact with the bacterial cell membrane. Superoxide anion oxidative stress is also one of the antibacterial causes (10). A graphene composite hydrogel was prepared by synthesizing silver/graphene composites of different mass ratios and then crosslinking with polyacrylic acid and polymethylidene bisacrylamide. When the mass ratio of silver to graphene in the hydrogel is $5: 1$, the hydrogel exhibits good mechanical properties as well as excellent antibacterial and biocompatibility. Due to the simple preparation and low cost of $\mathrm{GO}$, the antibacterial property of GO is expected to be widely used in environmental and clinical fields. However, different studies have found that GO dispersions and films not only inhibit the proliferation of bacteria, but also promote the growth of bacteria (11). It is believed that in the previous study, GO could play an antibacterial role, the source may be the pollutants left in the preparation process of $\mathrm{GO}$ or underestimate the concentration of $\mathrm{GO}$, and they use less dialysis after long-term dialysis (12). This shows that the preparation process and quality of GO will seriously affect the number of bacteria.

\section{Promote Cell Growth and Differentiation Graphene}

GO films can increase their growth ability by promoting the adhesion and proliferation of mammalian cells (13). Graphene has low cytotoxicity and can accelerate the differentiation of bone marrow mesenchymal stem cells (hMSCs) into osteoblasts, and its differentiation speed is similar to that of BMP-2. The surface of graphene is suitable for attachment of osteoblasts on its surface, and the attachment of osteoblasts is directly related to its cell activities such as proliferation and differentiation. It has also been found that graphene is beneficial to the mineralization of phosphate and calcium salts on its surface. , that is, the ability to form bone mineralization is excellent, and this series of cell activities can promote the formation of new bone on the surface of the implant. In addition, iPSCs can adhere and proliferate on the surface of GO and rGO, but rGO can inhibit the differentiation of iPSCs, while GO can promote its differentiation. Different surface properties of graphene are important factors regulating the growth and differentiation behavior of iPSCs (14).

Graphene has good biocompatibility, can promote the adhesion of hNSCs and induce its differentiation into neuronal cells instead of glial cells, and can significantly increase the number and average length of neurites. It is a potential neural interface material. Three-dimensional graphene foam neural stem cell scaffold material 3D-GFs can make neural stem cells adhere and proliferate on the surface of 3D-GFs, and can be further differentiated into astrocytes, especially neuronal cells (15). In addition, the birth of 3D printed graphene ink (graphene content up to 60\%) makes it flexible and safe to be applied in the biomedical field; in vivo experiments have confirmed that the graphene scaffold structure using the ink 3D printing has good biocompatibility; In vitro experiments have shown that graphene scaffolds do not affect stem cell survival, and can promote their continued division, proliferation and transformation into neuron-like cells (16).

\section{THE BIOSAFETY OF GRAPHENE}

Due to its excellent structural, mechanical, thermal, optical, electrical and biological properties, graphene can be used in many fields of biomedicine. However, the clinical application of any material must consider its potential biosafety.

The main component of graphene is carbon, which is one of the most basic elements in bioorganisms. Therefore, graphene materials have natural advantages in biomedical applications. Due to the different preparation processes, the physical and chemical properties of graphene and its derivative materials are different, so there are many ways to interact with biomolecules, cells, tissues and organs. At least current research shows that graphene is a carbon nanomaterial with good biocompatibility. 
Cytotoxicity is one of the important indicators for assessing the safety of biological materials. Graphene materials generally exhibit lower cytotoxicity. Although individual studies have found that GO exhibits a high hemolysis rate, the cytotoxicity of graphene materials is significantly reduced after modification with polyethylene glycol, chitosan, Tween, artificial peroxidase, dextran, and protein. Animal toxicity is another important indicator for assessing the biosafety of materials. Although graphene and its derivatives can be enriched in the lungs and exist for a long time, which leads to the formation of pulmonary edema and granuloma, which induces lung injury, but also polymers such as polyethylene glycol, dextran, chitosan, etc. Modification can significantly reduce toxicity in vivo. In addition, the presence of horseradish peroxidase, human myeloperoxidase, etc. in the organism can cause degradation of graphene materials, which can greatly reduce the biotoxicity of graphene materials, especially its long- term biotoxicity, making it safer and more effective for biomedical applications.

The great research progress of graphene in the field of biomedicine is inspiring, but we cannot deny the challenge. Due to the diversity of graphene-based materials and the complexity of biological systems, the mechanism of interaction between graphene and biomolecules, cells, tissues, organs and even organisms has not been thoroughly studied. Therefore, how to improve the biocompatibility and stability of graphene materials under the premise of maintaining the physical and chemical properties of graphene materials is an important research direction. In addition, we must know that graphene is not a "universal material", so it needs to be modified for each specific application environment to develop corresponding graphene-derived materials, which requires researchers with different academic backgrounds to work together.

\section{ARTICLE INFORMATION}

Author Affiliations: Division of Biology and Chemistry (DBC) (Clithy), The BASE, Chapel Hill, NC 27157, USA.

Author Contributions: Dr Clithy E. had full access to all of the data in the study and takes responsibility for the integrity of the data and the accuracy of the data analysis.

Study concept and design: Clithy.

Acquisition, analysis, or interpretation of data: Clithy.

Drafting of the manuscript: Clithy.
Critical revision of the manuscript for important intellectual content: Clithy.

Statistical analysis: N/A.

Obtained funding: N/A.

Administrative, technical, or material support:

Clithy.

Study supervision: Clithy.

Conflict of Interest Disclosures: Clithy E. declared no competing interests of this manuscript submitted for publication.

Funding/Support: Department support.
Role of the Funder/Sponsor: N/A.

How to Cite This Paper: Clithy E. Graphene: A promising candidate for biomedical use. Sci Insigt. 2019;29(3):52-57.

Digital Object Identifier (DOI): http://dx.doi.org/10.15354/si.19.re089.

Article Submission Information: Received, June 06, 2019; Revised: June 19, 2019; Accepted: July 02, 2019.

\section{REFERENCES}

1. Novoselov KS, Geim AK, Morozov SV, Jiang D, Zhang Y, Dubonos SV, Grigorieva IV, Firsov AA. Electric field effect in atomically thin carbon films. Science 2004; 306(5696):666-669.

2. Taniselass S, Md Arshad MK, Gopinath SCB. Current state of green reduction strategies: Solutionprocessed reduced graphene oxide for healthcare biodetection. Mater Sci Eng C Mater Biol Appl 2019; 96:904914.

3. Xu T, Zhang Z, Qu L. Graphenebased fibers: recent advances in preparation and application. Adv Mater 2019: 2019:e1901979.
4. Ren S, Lv L, Ma J, Lu H, Guo J, Li X, Dong G, Li J, Dang X. Slow-release lubrication effect of graphene oxide/poly(ethylene glycol) wrapped in chitosan/sodium glycerophosphate hydrogel applied on artificial joints. Mater Sci Eng C Mater Biol Appl 2019; 98:452-460.

5. Fathalipour S, Ataei B, Janati F. Aqueous suspension of biocompatible reduced graphene oxide- Au NPs composite as an effective recyclable catalyst in a Betti reaction. Mater Sci Eng C Mater Biol Appl 2019; 97:356366.
6. Colchester RJ, Alles EJ, Desjardins AE. A directional fibre optic ultrasound transmitter based on a reduced graphene oxide and polydimethylsiloxane composite. Appl Phys Lett 2019; 114(11):113505.

7. Morales DM, Barwe S, Vasile E, Andronescu C, Schuhmann W. Enhancing electrocatalytic activity by liquid-phase exfoliation of NiFe LDH intercalated with metal phthalocyanines in the presence of graphene. Chemphyschem. 2019 Jul 24. doi: 10.1002/cphc.201900577.

8. Tamayo Marín JA, Londoño SR, Del- 
gado J, Navia Porras DP, Valencia Zapata ME, Mina Hernandez JH, Valencia $\mathrm{CH}$, Grande Tovar CD. Biocompatible and antimicrobial electrospun membranes based on nanocomposites of chitosan/poly (vinyl alcohol)/graphene oxide. Int J Mol Sci 2019; 20(12). pii: E2987.

9. Fallatah $\mathrm{H}$, Elhaneid $\mathrm{M}$, Ali-Boucetta $H$, Overton TW, El Kadri $H$, Gkatzionis K. Antibacterial effect of graphene oxide (GO) nano-particles against Pseudomonas putida biofilm of variable age. Environ Sci Pollut Res Int 2019 Jun 27. doi: 10.1007/s11356-019-05688-9.

10. Oloyede HOB, Ajiboye HO, Salawu $\mathrm{MO}$, Ajiboye TO. Influence of oxidative stress on the antibacterial activity of betulin, betulinic acid and ursolic acid. Microb Pathog 2017; 111:338344.
11. Pan N, Wang Y, Ren X, Huang TS, Kim IS. Graphene oxide as a polymeric $\mathrm{N}$-halamine carrier and release platform: Highly-efficient, sustainedrelease antibacterial property and great storage stability. Mater Sci Eng C Mater Biol Appl 2019; 103:109877.

12. Goto Y, Yoshida N. Microbially reduced graphene oxide shows efficient electricity ecovery from artificial dialysis wastewater. J Gen Appl Microbiol 2017; 63(3):165-171.

13. Zhou M, Lozano N, Wychowaniec JK, Hodgkinson T, Richardson SM, Kostarelos K, Hoyland JA. Graphene oxide: a growth factor delivery carrier to enhance chondrogenic differentiation of human mesenchymal stem cells in 3D hydrogels. Acta Biomater 2019; pii: S1742-7061(19)30516-1.

14. Saburi $E$, Islami $M$, Hosseinzadeh $S$, Moghadam AS, Mansour RN,
Azadian E, Joneidi Z, Nikpoor AR, Ghadiani MH, Khodaii Z, Ardeshirylajimi A. In vitro osteogenic differentiation potential of the human induced pluripotent stem cells augments when grown on Graphene oxide-modified nanofibers. Gene 2019; 696:72-79.

15. Sakai K, Teshima TF, Nakashima $H$, Ueno Y. Graphene-based neuron encapsulation with controlled axonal outgrowth. Nanoscale 2019; 11(28):13249-13259.

16. Qin EC, Kandel ME, Liamas E, Shah TB, Kim C, Kaufman CD, Zhang ZJ, Popescu G, Gillette MU, Leckband DE, Kong H. Graphene oxide substrates with $\mathrm{N}$-cadherin stimulates neuronal growth and intracellular transport. Acta Biomater 2019; 90:412-423. 